\title{
Journal of Health \& Medical

\section{A Randomised Single-Blinded Controlled Trial on the Effectiveness of Brief Advice on Smoking Cessation among Tertiary Students in Malaysia}

De Silva WDAS ${ }^{1}$, Awang $\mathbf{R}^{1}$, Samsudeen $\mathbf{S}^{1}$ and Hanna $\mathbf{F}^{2^{*}}$

${ }^{1}$ National Poisons Centre of Malaysia, University Sains Malaysia

${ }^{2}$ Program of Public Health, Department of Health Sciences, College of Arts and Sciences, Qatar University, Qatar

"Corresponding author: Hanna F, Program of Public Health, Department of Health Sciences, College of Arts and Sciences, Qatar University, Qatar, Tel:+974 4403-451; E-mail: fhanna@qu.edu.qa

Received date: February 06, 2016; Accepted date: February 23, 2016; Published date: February 28, 2016

Copyright: @ 2016 De Silva WDAS, et al. This is an open-access article distributed under the terms of the Creative Commons Attribution License, which permits unrestricted use, distribution, and reproduction in any medium, provided the original author and source are credited.

\section{Abstract}

Introduction: Tobacco smoking, a habitual behavior, is addictive and detrimental to health. Quitting requires personal abilities and environmental opportunities and therefore, improving these abilities and opportunities will undoubtedly act on smokers' motivation to quit.

Methods: A prospective single-blinded randomized controlled interventional study was conducted among first year undergraduate students in Malaysia. A total of eighty smokers were randomly allocated to a control or intervention groups (40/40). Randomization remained concealed from research personnel. All participants were followed up for six months to evaluate abstinence.

Results: Quit line enrolment rate of the intervention group was 55\% (22) compared to $7.5 \%(3)$ in the control $(\mathrm{P}<$ $0.00195 \% \mathrm{Cl} 30.1-64.9)$. In the intervention group $27 \%$ (6) sustained quitting for six months compared to none in the control group.

Conclusion: This study has shown that brief advice for smoking cessation is more effective than an information leaflet alone to promote quitting and that to maintain abstinence quit line follow up is necessary. Larger samples size and longer follow up studies are needed to further confirm these findings.

Keywords: Brief advice; Smoking cessation; Tobacco smoking; Quit line

\section{Introduction}

Non communicable diseases (NCDs) such as cardiovascular diseases, diabetes, cancers and chronic respiratory diseases had caused nearly two third (36 million) of the 57 million global deaths in 2008 [1]. Smoking contributes extensively to mortality from NCDs, including CVDs, cancer and chronic respiratory diseases. Moreover, nearly 6 million people die from tobacco use each year. Smoking is estimated to cause about $71 \%$ of lung cancer, $42 \%$ of chronic respiratory disease and nearly $10 \%$ of cardiovascular disease [1]. In the ASEAN region $30 \%$ of adults are reportedly current smokers [2].

Tobacco is the second biggest cause of all deaths and disabilities from NCDs in Malaysia. National Health Morbidity Survey (NHMS) 2006 revealed that diseases related to smoking had contributed to $15 \%$ of morbidity in government hospitals and $35 \%$ of all hospital mortality in 2006 [3]. The Global Adults Tobacco Survey Malaysia (GATS) 2011 revealed that the smoking prevalence among adults was $43.9 \%$ among men and $1 \%$ among women with overall $23.1 \%$ [4]. World Health Statistics 2010 reveals that prevalence of smoking any tobacco product among over 15 years old males was $52.6 \%$ and $2.6 \%$ in females in Malaysia. The above report also indicated that the prevalence of tobacco use among 13 - 15 years old adolescent males and females was $35.1 \%$ and $9.4 \%$, respectively, for the period $2000-2009$ [5].
Khor's study on tobacco use among female college and university students in Kuala Lumpur, Malaysia in 2005 revealed that $21.3 \%$ of the students had ever smoked and $4.3 \%$ were currently smoking. Most of them were smoking less than 10 cigarettes daily [6].

Though the prevalence of smoking and its association with gender, ethnicity and socioeconomic backgrounds were investigated, the cognitive aspect influencing smoking was scarcely examined in the previous studies, except for the one by Foong Kin [7] and the other by Lim et al. [8] on knowledge and attitudes on tobacco.

As Rothschild et al explains, smoking behavior is influenced by mediators or determinants, different cognitions and environmental factors. The mediators include motivation, abilities and opportunities $[9,10]$.

The most popular theories used for the development of smoking behavior change intervention are the Trans Theoretical Model (TTM) and its stages of change concepts. According to John et al. [11] effective smoking prevention interventions are based on the National Cancer Institute's Five A's model, the Agency for Health Research and Quality guidelines and the Trans theoretical Model (TTM) of behavioral change. Five A's model is based on asking about smoking behavior, advice, assess, assist and arrange follow up. [12] The TTM facilitates application of the intervention to match the smoker's needs [13]. The level of addiction to nicotine is an indication of cessation success [14].

Meanwhile, $21.5 \%$ of the population is exposed to secondhand smoke in public places in Malaysia [3]. A study by Jiyeon et al. revealed 
significantly high particulate matter levels due to second hand smoking in public places [15].

Researchers have been relentlessly studying at the effect of brief advice on smoking cessation rate. A study by Hjalmarson and Boethius showed this rate to be $25 \%$ among those who received brief advice [16]. Moreover, brief advice given by health professionals around three minutes has been revealed to decrease the proportion of people smoking by $2 \%$ when compared to those who did not get any advice [17]. More recently, randomized controlled trials on smoking cessation have been conducted in low-middle income countries settings. A Hong Kong study on the duration of abstinence that used cash incentive as a reward for quitting showed that a brief telephone advice was positively associated with abstinence while short service messaging and no intervention had no effect [18]. A new study protocol by Indian researchers on the effect of brief advice, including training in craving control, has been published [19]. The above randomized controlled trial will attempt to show the effectiveness of a brief advice on quitting smoking, conducted by "trained health professionals".

The aim of our study is to compare the effectiveness of translating intention to quit smoking into action by enhancing motivation through a face-to-face "brief advice" and by a Quit line leaflet. "Brief advice" is a short intervention (usually from 30 seconds to 3 minutes) delivered opportunistically in relation to a client's reason for seeking help. Brief advice is less in depth and more informal than a brief intervention and usually involves giving information about the importance of behaviour change and simple advice to support behaviour change [20].

The objectives of the study were:

To evaluate the effectiveness of Brief Advice on motivating smokers to quit smoking.

To assess the effectiveness of sustaining smoking cessation facilitated by USM Quit line.

\section{Methods}

\section{Study design}

This randomized control trial.

Phase 1: The University Medical Officers screened all the students for their smoking status during the medical examination. Smoking status was self-declared by the students.

Phase 2: A prospective randomized control trial with two parallel groups, one to receive brief advice and the other to receive only a conventional leaflet with basic information on smoking risk, importance of quitting and the services provided by Quit Line. Researchers were blinded to the randomization.

\section{Reference population}

Tertiary students in Malaysian Universities.

\section{Study population and recruitment}

Sampling frame: Male and female undergraduate students enrolled in the main campus of the University Sains Malaysia.
Inclusion criteria: The undergraduate students, who were residents of Malaysia, enrolled in the main campus of the USM during 2011 and 2012.

\section{Sample size}

Phase I: All male and female undergraduate students in the main campus of the Universiti Sains Malaysia were screened for their smoking status during the routine medical examinations at the Medical Centre.

Phase II: During six months prospective study involving independent cases and controls with 1 control(s) per case, to detect a $25 \%$ quit rate with a power of $80 \%$, minimum subjects required to each group is 40 . The Type I error probability associated with this test of this null hypothesis was 0.05 . The total sample size was $40 \times 2=80$ (P\&S calculator).

\section{Study process}

Intervention on brief advice was based on asking about smoking habit, explaining the disadvantages of smoking, advice on benefit of stopping, assess the willingness to quit and level of nicotine addiction and opportunities available through USM-quit line to assist in the quitting process. Control group received only a printed A4 size paper with basic information on risk of smoking and importance of quitting and the services provided by Quit Line. No other advice was given to them.

\section{Data collection}

A structured pre tested self-administered questionnaire was used for data collection. Questionnaire consisted of socio-demographic data, smoking practices, intention to quit, failed previous attempts, trigger for smoking and reasons for the intention to quit. The level of addiction to nicotine was assessed by the Fagerstrom nicotine tolerance questionnaire.

$\begin{array}{lc}\text { Total score } & \text { Level of dependence } \\ 0-3 \text { points } & \text { Low } \\ 4-6 \text { points } & \text { Medium } \\ 7-10 \text { points } & \text { High }\end{array}$

The questionnaire was administered in the English language. Identification of the persons was confirmed by checking the National Identity card number and the student identity number.

Ethics approval was obtained from the Ethical Review Committee of the University Sains Malaysia. Initially the study procedure was explained to the participants and an information sheet was given them to read and understand.

Confidentiality was ensured by concealing the identity and personal data to investigators. The respondents were given a case number only. After clarification of any doubts, written informed consent was obtained from the respondents to participate in the study and to register them in the Quit line and for follow up.

\section{Data analysis}

Quantitative data was analyzed using descriptive statistics. Primary analysis included the number and proportions experiencing an event. Mean estimation of proportions and $95 \%$ confidence interval was 
Page 3 of 4

calculated to ascertain the magnitude and the direction of the effect. Chi-square test was used to compare the categorical variables and $\mathrm{T}$ test was used for comparison of continuous variables. SPSS 15 was used for data analysis.

\section{Results}

All the female students were declared as non-smokers. Among the total number of male respondents $(n=80)$, Brief advice was received by 40 in the intervention group and USM Quit line Information leaflet was given to 40 in the Control group.

The basic characteristics of the two groups are described in Table 1.

\begin{tabular}{|l|l|l|}
\hline & Intervention $(\mathbf{n}=\mathbf{4 0})$ & Control $\mathbf{n}=\mathbf{4 0})$ \\
\hline $\begin{array}{l}\text { Mean age of the respondents } \\
\text { (years) }\end{array}$ & 21.2 & 22.6 \\
\hline $\begin{array}{l}\text { Mean duration of smoking } \\
\text { (years) }\end{array}$ & 4.4 & 6.7 \\
\hline Previous quit attempts & $4(10 \%)$ & $7(17.5 \%)$ \\
\hline No attempts & $10(25 \%)$ & $9(22.5 \%)$ \\
\hline Once $(\mathbf{n}=40)$ & $11(27.5 \%)$ & $11(27.5 \%)$ \\
\hline Twice & $15(37.5 \%)$ & $13(32.5 \%)$ \\
\hline \multicolumn{1}{|c|}{3 times } & $1(2.5 \%)$ & $4(10 \%)$ \\
\hline Readiness to quit smoking & $16(40 \%)$ & $10(25 \%)$ \\
\hline Not ready & $18(45 \%)$ & $11(27.5 \%)$ \\
\hline Not sure & $5(12.5 \%)$ & $15(37.5 \%)$ \\
\hline Ready & & \\
\hline Very ready & & \\
\hline
\end{tabular}

Table 1: The baseline characteristics of the two groups.

Among those who registered in the USM quitline, 13 (59\%) had low nicotine dependence while only $2(9 \%)$ had high nicotine dependence. Among the 22 in the intervention group who received Brief Advice 06 (27\%) respondents continued to quit smoking up to 6 months compared to none in the control.

Following the brief advice, 22 (55\%) students from the intervention group registered in the USM quit line compared to $03(7.5 \%)$ the control group which was statistically significant $(\mathrm{P}<0.001)$ (Table 2$)$.

\begin{tabular}{|c|c|c|}
\hline \multicolumn{3}{|l|}{ Enrollment in the USM Quit line } \\
\hline $\begin{array}{l}\text { Intervention }(n= \\
40)\end{array}$ & Control $(n=40)$ & $p$ value \\
\hline $22(55 \%)$ & $3(7.5 \%)$ & $\begin{array}{l}\mathrm{P}<0.001(95 \% \mathrm{Cl} \\
30-64)\end{array}$ \\
\hline \multicolumn{3}{|l|}{ Sustained quitting for six months } \\
\hline $06(27 \%)$ & $00(0 \%)$ & \\
\hline
\end{tabular}

Table 2: Outcome of the intervention.

\section{Discussion}

Our findings show that brief advice to quit smoking is more effective than an information leaflet alone. The cessation rate of $27 \%$ in this study is comparable with the cessation rate of $25 \%$ among those who received brief advice in a study by Hjalmarso [16]. Among the 22 in the intervention group who received Brief Advice, 6 (27\%) respondents continued to quit smoking up to 6 months compared to none in the control. These findings are also consistent with similar study one quitting conducted by a Hong Kong researchers that showed that telephone-based advice was positively associated with abstinence in comparison to advice via short messaging service or no advice at all [18].

Furthermore, our results showed that 04 (18\%) respondents had set new quit dates in-spite of having set prior quit dates and having preparatory sessions. During the six months, harm reduction was also evident as they have reduced the number of cigarettes smoked by $40 \%$. Interestingly, 07 (32\%) had set quit dates but they did not respond to the subsequent quit line calls. Meanwhile 05 (23\%) had registered in quit line they did not respond to quit line calls at all.

Among the controls 3 respondents (7.5\%) had quitted smoking but no one abstained for six months but harm reduction was evident by the reduction of number of cigarettes smoked by $30 \%$.

Smoking behavior is influenced by mediators, different cognitions and environmental factors. As Rothschild et al. importance explains, the mediators include motivation, abilities and opportunities. The relative and underlying beliefs of these determinants vary among different populations and between individuals within the same population depending on their personal, social and environmental circumstances [12]. In this study, following the brief advice, 22 (55\%) students from the intervention group registered in the USM quit line compared to $03(7.5 \%)$ in the control group clearly indicate their motivation level to quit.

Cahill et al. reveals (by the Cochrane review) stage based self-help interventions and individual counseling to be neither more nor less effective than the non-staged based interventions provided at a similar intensity USM Quit line is based on 5As and TTM. On par with this, among the 22 in the intervention group who received Brief Advice06 (27\%) respondents continued to quit smoking up to 6 months compared to none in the control. The remainder of respondents were fitting into the various stages of TTM.

Habitual complex behaviors depend very much on personal abilities and environmental opportunities as explained by Gollwitzer PM, the theories that improve people's abilities and opportunities will effectively act on their motivation. Such action oriented self-regulatory models focus specifically on the cognitive mechanisms involved in translating an intention to perform a particular behavior into action as evidenced by the outcomes in the current study.

The uniqueness of our study is that we used a simple face-to-face brief advice without the use of formal professional expertise. This seems to be more applicable and practical exercise, at least in resource poor settings.

\section{Limitations}

Smoking status was declared by the respondents. Confirmation through the biological validation was not done in this study due to financial constraints. Moreover, a larger sample size and a longer 
Citation: De Silva WDAS, Awang R, Samsudeen S, Hanna F (2016) A Randomised Single-Blinded Controlled Trial on the Effectiveness of Brief Advice on Smoking Cessation among Tertiary Students in Malaysia. J Health Med Inform 7: 217. doi:10.4172/2157-7420.1000217

Page 4 of 4

follow-up period would have allowed healthier numbers in the subgroups which would have further improved the results. However, this is also strength as our study showed a significant gap between the intervention and the control group in relation to the outcome of interest and we can, more or less, predict that a larger sample with longer follow-up may show yet more substantial outcome.

\section{Conclusion}

"Brief advice for smoking cessation"can be more effective than an information leaflet alone in promoting smokers to quit smoking. For the abstinence, quit line follow up is vital. More research with larger sample size should be carried out to test the efficacy of this new approah to encourage young people to quit smoking that can be extended to other groups and communities as well.

Acknowledgement

We would like to acknowledge the Asian Collaboration for Excellence in Non-Communicable Diseases (ASCEND) Research Training Program Funded by the Fogarty International Centre, US National Institutes of Health Award Number: D43TW008332 for all the technical support and the financial support given to me to make the study a success. My special thanks deserves to Prof Brian Oldenberg Director, ASCEND for the kind advise and directions given to me and the project staff. My sincere thanks also to the staff of the USM quit line service, the staff of the Medical Centre of the University Sains Malaysia and Prof. Wah Yun Low, my International Mentor.

\section{References}

1. The WHO global status report on non-communicable diseases 2010 .

2. http://seatca.org/dmdocuments/ASEAN-Tobacco-Control-Report-2012.

3. The Third National Health and Morbidity Survey III- Ministry of Health, Malaysia. 2007.

4. www.who.int/tobacco/.../survey/gats/malaysia_fact_sheet_2011.pd

5. www.who.int/gho/world_health_statistics

6. Khor YL (2005) Factors Associated With Tobacco Use Among Female College and University Students in Kuala Lumpur, Malaysia. National Poison Centre, Universiti Sains Malaysia, Penang.
7. Foong Kin (2008) smoking in Girls and Young Women in Malaysia. SEATCA.

8. Lim K, Sumarni MG, Amal NM, Hanjeet K, Wan Rozita WM (2009) Tobacco use, knowledge and attitude among Malaysians age 18 and above. Tropical Biomedicine 26: 92-99.

9. Rothchild ML (1999) A conceptual framework for the management of Public Health and Social Issue Behaviours. J Mark 63: 24-37.

10. Golwitzer PM (1999) Implementation intentions: strong effects of simple plans. Am Psychol 54: 493-450.

11. John, Robert AN (2003) Tobacco cessation in primary care. Clinical Medicine and Research 1: 205-206.

12. http://www.sharinginhealth.ca/healthy_choices/change/five_As.html

13. Prochaska, Velicer WF, Fava JL, Rossi JS, Tsoh JY (2001) Stage based expert system intervention for smoking cessation. Addictive Behaviours 26: $583-602$

14. Heatherton TF, Kozlowski LT, Frecker RC, Fagerstrom KO (1991) The Fagerström Test for Nicotine Dependence: a revision of the Fagerström Tolerance Questionnaire. Br J Addict 86: 1119-1127.

15. Jiyeon L, Lim S, Lee K, Guo X, Kamath R, et al. (2010) Secondhand smoke exposure in seven Asian countries. International journal of hygiene and environmental health 213: 348-351.

16. Hijalmarson A, Boethius G (2007) The effectiveness of brief advice and extended smoking cessation counseling programs when implemented routinely in hospitals. Preventive Medicine 45: 202-207.

17. Clinical Audit, Supporting smoking cessation.

18. Chan SS, Wong DC, Cheung YT, Leung DY, Lau L, et al. (2015) A block randomized controlled trial of a brief smoking cessation counselling and advice through short message service on participants who joined the Quit to Win Contest in Hong Kong Health Educ Res 30: 609-621.

19. Sarkar BK, Shahab L, Arora M, Lorencatto F, Reddy KS, et al. (2014) A cluster randomized controlled trial of a brief tobacco cessation intervention for low-income communities in India: study protocol. Addiction 109: 371-378.

20. Powell K, Thurston M (2008) Commissioning training for behaviour change interventions: Evidence and best practice in delivery. 